SUBmitted TO ApJ

Preprint typeset using LATEX style emulateapj v. 5/2/11

\title{
THE ROTATION OF THE HOT GAS AROUND THE MILKY WAY
}

\author{
Edmund J. Hodges-Kluck ${ }^{1}$, Matthew J. Miller ${ }^{1} \&$ Joel N. Bregman ${ }^{1}$ \\ submitted to ApJ
}

\begin{abstract}
The hot gaseous halos of galaxies likely contain a large amount of mass and are an integral part of galaxy formation and evolution. The Milky Way has a $2 \times 10^{6} \mathrm{~K}$ halo that is detected in emission and by absorption in the O VII resonance line against bright background AGNs, and for which the best current model is an extended spherical distribution. Using XMM-Newton RGS data, we measure the Doppler shifts of the O VII absorption-line centroids toward an ensemble of AGNs. These Doppler shifts constrain the dynamics of the hot halo, ruling out a stationary halo at about $3 \sigma$ and a corotating halo at $2 \sigma$, and leading to a best-fit rotational velocity $v_{\phi}=183 \pm 41 \mathrm{~km} \mathrm{~s}^{-1}$ for an extended halo model. These results suggest that the hot gas rotates and that it contains an amount of angular momentum comparable to that in the stellar disk. We examined the possibility of a model with a kinematically distinct disk and spherical halo. To be consistent with the emission-line X-ray data the disk must contribute less than $10 \%$ of the column density, implying that the Doppler shifts probe motion in the extended hot halo.
\end{abstract}

Subject headings: Galaxy: halo — Galaxy: kinematics and dynamics — Galaxy: structure

\section{INTRODUCTION}

A basic prediction of $\Lambda \mathrm{CDM}$ galaxy-formation models is the existence of a hot $\left(10^{6}-10^{7} \mathrm{~K}\right)$ halo of gas accreted from the intergalactic medium around Milky Way-sized galaxies (extending to the virial radius), which forms as infalling gas is heated to the virial temperature at an accretion shock (e.g., White \& Frenk 1991). These halos may provide most of the fuel for long-term star formation in these galaxies (Crain et al. 2010; Joung et al. 2012), but their predicted properties are sensitive to the input physics, which can be constrained by the measurable properties of the gas.

Based on work over the past several years, we know that these extended halos exist, including around the Milky Way (Anderson \& Bregman 2011; Anderson et al. 2013; Miller \& Bregman 2015). The extent and luminosity of the hot gas implies that it has a similar mass to the stellar disk, and therefore could play an important role in galaxy evolution. Thus, it is important to measure the properties of the hot gas beyond mass and temperature (such as metallicity and density or velocity structure). However, hot halos are faint and the measurable X-ray luminosity can be dominated by stellar feedback ejecta near the disk (Li \& Wang 2013), which makes these measurements difficult.

Only in the Milky Way can one measure the structure, temperature, metallicity, and kinematics of the hot gas through emission and absorption lines (Nicastro et al. 2002; Paerels \& Kahn 2003; McKernan et al. 2004; Yao \& Wang 2005; Williams et al. 2005; Fang et al. 2006; Williams et al. 2006, 2007; Yao et al. 2009b; Henley \& Shelton 2012; Gupta et al. 2012), but kinematic constraints from prior studies (Bregman \& Lloyd-Davies 2007; Fang et al. 2015) are weak. Recent developments in the calibration of the X-ray grating spectrometers and the accumulation

\footnotetext{
hodgeskl@umich.edu

${ }^{1}$ Department of Astronomy, University of Michigan, Ann Arbor, MI 48109
}

of multiple high quality data sets for individual objects have made it possible to determine line centroids to an accuracy of tens of $\mathrm{kms}^{-1}$, which enables us to improve the constraints on the kinematics of the gas by measuring Doppler shifts in lines that trace the hot gas. The $\lambda 21.602 \AA$ resonance O VII absorption line (Drake 1988) is the best candidate, since it is sensitive to temperatures of $10^{5.5}-10^{6.3} \mathrm{~K}$ (which includes much of the Galactic coronal gas) and it is detected at zero redshift towards a large number of background continuum sources (e.g., Fang et al. 2015). The emission lines produced by the same species are useful for determining the structure and temperature of the hot gas (Henley \& Shelton 2012; Miller \& Bregman 2015), but they are too faint for high resolution spectroscopy. In this paper we constrain, for the first time, the radial and azimuthal velocity of the hot gas by measuring the Doppler shifts in O VII lines detected towards bright sources outside the disk of the Milky Way.

\section{OBSERVATIONS AND DATA ANALYSIS}

\subsection{Sample and Reduction}

To measure the global velocity of the million-degree gas around the Galaxy, one needs to measure Doppler shifts towards a range of sources across the sky in lines sensitive to this temperature. This gas is detected in X-ray emission and absorption, but the emission lines are far too faint for a focused grating observation. X-ray imaging CCDs measure the energies of incoming photons and are thus also low resolution spectrometers, but their spectral resolution is far too low to measure Doppler shifts of tens of $\mathrm{km} \mathrm{s}^{-1}$. The only instruments capable of this accuracy are the Chandra Low/High-Energy Transmission Grating (LETG/HETG) Spectrometers and the XMMNewton Reflection Grating Spectrometer (RGS), and the $21.602 \AA \mathrm{O}$ VII line is the only line that probes the relevant temperatures and is detected at $z=0$ towards many background continuum sources. The OVIII line at $18.96 \AA$ probes slightly hotter gas and is only detected towards a 
few objects.

Our initial sample included all archival LETG and RGS data sets where the line has been detected in the literature. The LETG has modestly better spectral resolution at $21.6 \AA(R=400)$ than the $\operatorname{RGS}(R=325)$, but it only has a third of the effective area at this wavelength $\left(15 \mathrm{~cm}^{2}\right.$ for the LETG, $45 \mathrm{~cm}^{2}$ for the RGS). In addition, unlike the LETG the RGS is always on (it has a dedicated telescope, whereas the gratings must be moved into the focal plane on Chandra), and thus has accumulated many more spectra. These factors lead to many more detected O VII lines in the RGS, so we only use the LETG data towards several calibration sources as a check on the wavelength solution (see below).

Our analysis sample includes 37 known O VII absorbers at $z=0$ with RGS data (Nicastro et al. 2002; Fang et al. 2002; McKernan et al. 2004; Williams et al. 2005; Yao \& Wang 2005; Fang et al. 2006; Williams et al. 2006, 2007; Yao et al. 2009b; Gupta et al. 2012; Fang et al. 2015) (Table 11). These include AGNs as well as several X-ray binaries in the Milky Way's halo and Magellanic Clouds. We tried to include all sources known to be outside the disk with reported absorption lines, but we excluded three sources: NGC 3783, PKS 2005 - 489, and Swift J1753.5 - 0127. NGC 3783 has an intrinsic oxygen line with a P Cygni profile where we cannot disentangle the Galactic line, PKS 2005 - 489 has a broad line that suggests blending or a non-Galactic origin, and the line in Swift J1753.5 - 0127 is only detected in two of four high $S / N$ exposures. We include NGC $5408 \mathrm{X}-1$ (which is an X-ray binary, not an AGN) and NGC 4051, but these systems have redshifts smaller than $1000 \mathrm{~km} \mathrm{~s}^{-1}$ so the lines may be intrinsic. NGC 4051 and MCG-6-30-15 also have known outflows; as they have O VII lines attributed to the Galaxy in some prior studies (e.g., Fang et al. 2015) we include them here, but we show below that excluding them does not strongly change our results.

The data for each target were reprocessed using standard methods in the XMM-Newton Science Analysis Software (SAS v14.0.0) with the appropriate calibration files. This included excluding hot, cold, and "cool" pixels, and data from periods when the background count rate exceeds $3 \sigma$ from the mean. We applied the (default) empirical correction for the Sun angle of the spacecraft and its heliocentric motion (de Vries et al. 2015). We used the highest precision coordinates available rather than the proposal coordinates, which improves the accuracy of the wavelength scale. For each object, we merged the first-order RGS1 spectra and response matrices into a "stacked" spectrum. Standard processing resamples the data from native bins (about $0.011 \AA$ at $21.6 \AA$ ) into a user-specified bin size. We binned the data to $0.02 \AA$ (one resolution element is about $0.055 \AA$ ). This resampling causes small but stochastic changes in the bin assignment for some events, leading to variation under the same protocols, which we quantify by processing each object ten times in the same way.

\subsection{Velocity Measurements}

To measure the Doppler shifts, we fit a model consisting of a power law and an absorption line to the spectrum in the 21-22 $\AA$ bandpass using XSPEC v12.9.0 Arnaud
1996). We exclude an instrumental artifact between $21.75-21.85 \AA$ in each spectrum, and in several spectra there are one or more bad channels in the bandpass that we also exclude2. The parameters of the absorption-line model include the line centroid, the line width, and the line strength, but we fix the line width at the instrumental line-profile width because we do not expect detectable line broadening, an assumption we validated in the brightest sources. The best-fit centroid is converted to a velocity using the best-fit line energy from a stacked spectrum of Capella as a reference, corrected for the radial velocity of the star (described below). We measured the velocity for each of the ten stacked spectra per object, and we report the mean value with its $1 \sigma$ uncertainty in Table 1, including the resampling uncertainty.

\subsection{Wavelength Scale Accuracy}

The systematic uncertainty in the wavelength scale limits the accuracy of our measurements, and recent improvements in the calibration of the wavelength scale (de Vries et al. 2015) and multiple high $S / N$ spectra for the objects in our sample are largely what enable this study. Here we show the accuracy of the wavelength solution for the protocols we adopt and briefly describe the sources of uncertainty and their magnitudes.

We created spectra for the active stars Capella and HR 1099 following the protocols above, then measured the (emission) line centroids for strong, mostly unblended lines, and compared them to their laboratory rest wavelengths (Figure 1). We accounted for the radial velocity of each star $\left(+29.2 \mathrm{~km} \mathrm{~s}^{-1}\right.$ for Capella and $-15.3 \mathrm{~km} \mathrm{~s}^{-1}$ for HR 1099; Karatas et al. 2004). We find no systematic offset in the $5-30 \AA$ bandpass or change over time. The wavelength offsets in O VII $\lambda 21.602 \AA$ for stacked spectra are $\Delta \lambda=0.0 \pm 0.8 \mathrm{m \AA}$ (Capella) and $0.7 \pm 1.6 \mathrm{~m} \AA$ (HR 1099). These correspond to $v=0 \pm 11$ and $-7 \pm 22 \mathrm{kms}^{-1}$. To verify that stacking does not introduce artificial offsets, we also measured centroids in each individual exposure (16 for Capella and 14 for HR 1099) and computed the weighted mean. The offsets are consistent with the stacked spectrum: $v=-8 \pm 26$ and $-3 \pm 28 \mathrm{kms}^{-1}$ for Capella and HR 1099 .

We also checked the wavelength solution against the LETG and HETG data for these stars. We reduced te data using the Chandra Interactive Analysis of Observations software (CIAO v4.7), and we found good agreement with spectra from the reduced data available through the TGCat project (Huenemoerder et al. 2011). We co-added the \pm first-order spectra and stacked all observations. The LETG offsets are $v=1 \pm 22 \mathrm{~km} \mathrm{~s}^{-1}$ (Capella) and $v=10 \pm 26 \mathrm{kms}^{-1}$ (HR 1099), whereas the HETG Medium Energy Grating offsets are $v=-3 \pm$ $21 \mathrm{~km} \mathrm{~s}^{-1}$ (Capella) and $v=-22 \pm 38 \mathrm{~km} \mathrm{~s}^{-1}$ (HR 1099). These results agree with the RGS and the laboratory wavelength (Figure 1). We also compared velocity measurements between the RGS and LETG in the four brightest quasars (Rasmussen et al.|2003; Williams et al. 2005; Bregman \& Llovd-Davies 2007; Rasmussen et al. 2007; Hagihara et al. 2010; Fang et al. 2015). The LETG and RGS centroids agree to within the $1 \sigma$ error bar.

\footnotetext{
2 see http://xmm.esac.esa.int/external/xmm_user_support/documentation/uh
} 

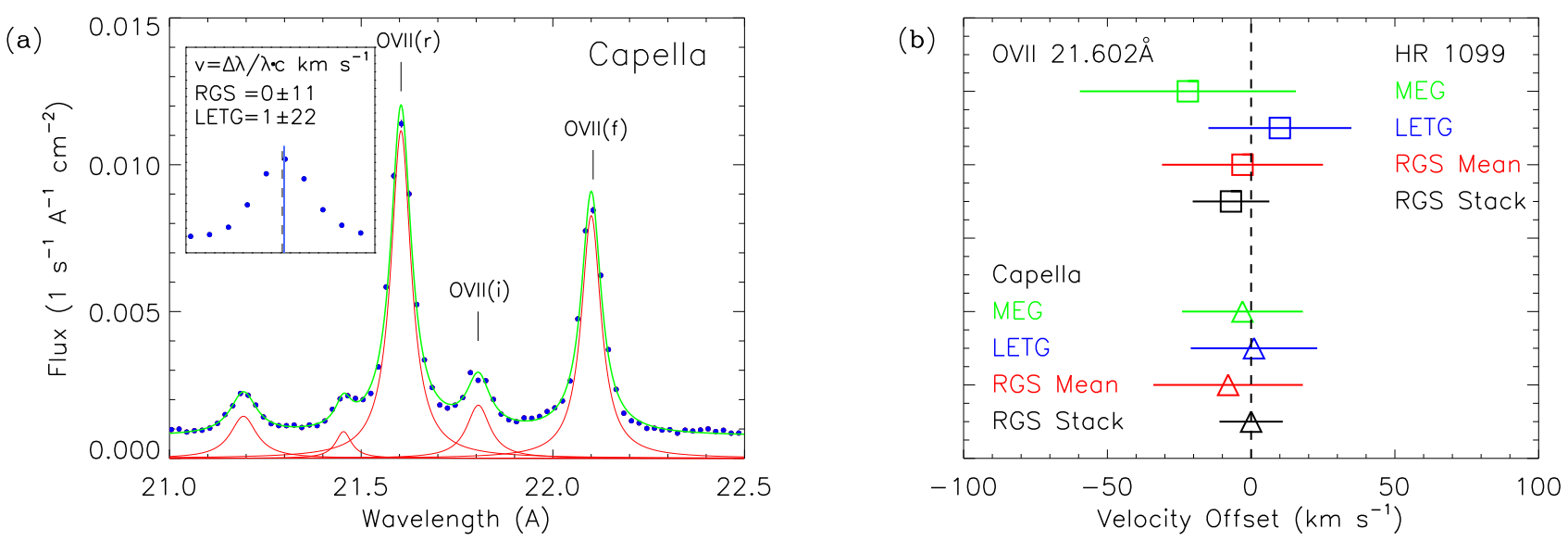

Figure 1. (a) The stacked RGS spectrum for Capella shows that the reduction protocols achieve an accurate wavelength solution (inset), and that averaging multiple data sets substantially reduces the systematic scatter in the wavelength grid. (b) Our measurements of the O VII emission line centroid relative to the $21.602 \AA$ rest wavelength in three instruments and two stars show that there are no serious cross-instrument issues with the wavelength grid. In each case, we accounted for the radial velocity of the stars.

(a)

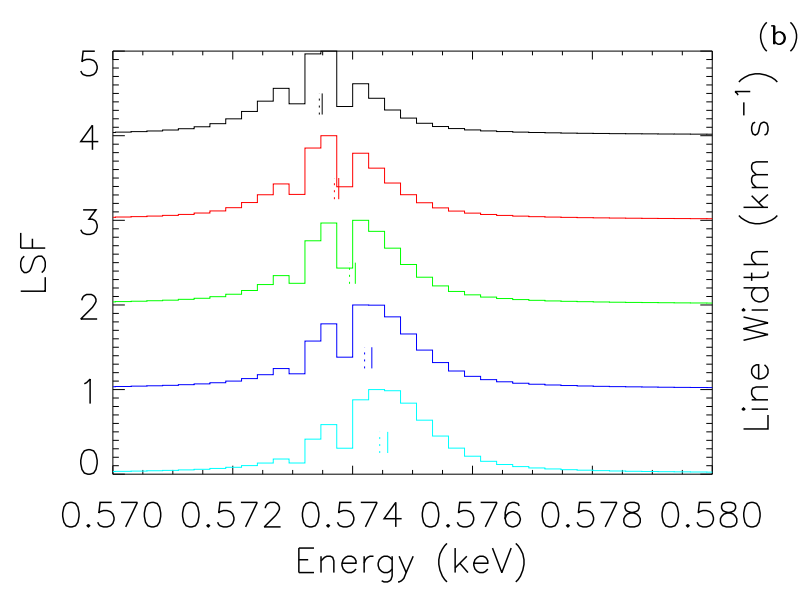

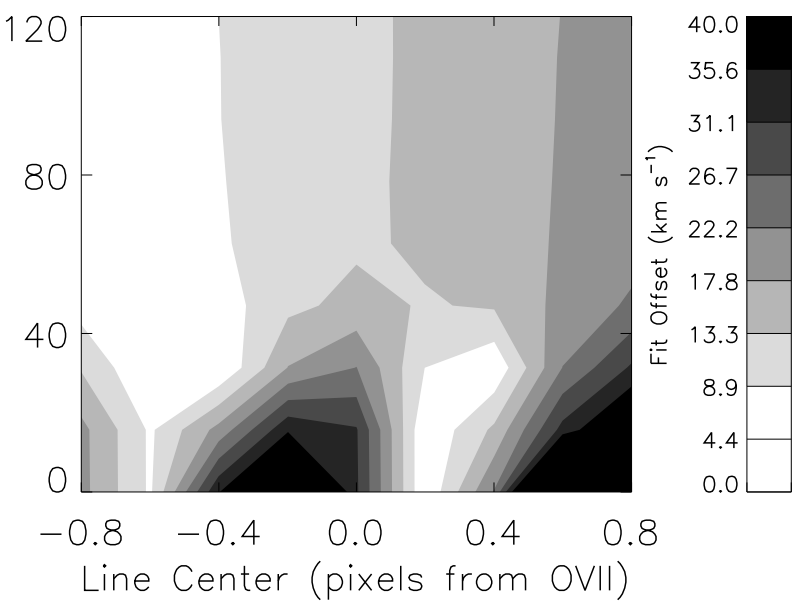

Figure 2. Bad columns near the O VII line can cause a systematic error in the centroid. (a) The difference between the nominal line center (dashed vertical tick) and the measured centroid (solid vertical tick) is a function of the true centroid. (b) The magnitude of this offset is plotted as a function of intrinsic line width and the nominal line centroid for Xspec simulations. For lines with an intrinsic width of $40 \mathrm{~km} \mathrm{~s}^{-1}$ or more, the typical error is about $15 \mathrm{~km} \mathrm{~s}^{-1}$.

These results show that the wavelength scale is sufficiently accurate for our measurement, but it is important to note that there is a substantial systematic scatter that makes individual observations unreliable, and also that there will be systematic differences between our measurements and those reported for the same data using an earlier version of SAS or using incorrect source coordinates.

Different observations of the same object have a systematic scatter of $\sim 5 \mathrm{~m} \AA$ in the wavelength solution around the true mean value. This corresponds to $70 \mathrm{~km} \mathrm{~s}^{-1}$ at $21.602 \AA$, and leads to the standard quoted systematic uncertainty of $100 \mathrm{~km} \mathrm{~s}^{-1}$. The reason for this scatter is not clear, but about $3 \mathrm{~m} \AA$ could be explained by limits in the pointing accuracy of the telescope (de Vries et al. 2015). In any case, the scatter is normally

3 see the XMM-Newton Users' Handbook at https://heasarc.gsfc.nasa.gov/docs/xmm/uhb/rgs.html distributed (based on measurements from many exposures of calibration stars such as Capella), and can thus be strongly mitigated with multiple observations of the same source. To reduce the scatter to within $20 \mathrm{~km} \mathrm{~s}^{-1}$ requires 15-20 independent spectra (assuming equal $S / N$ and a $\sigma=5 \mathrm{~m} \AA$ ). The sources in our sample have between 2-60 observations, and at the low end the reported $1 \sigma$ statistical errors $\left(200 \mathrm{~km} \mathrm{~s}^{-1}\right.$ or more) are much larger than a $70 \mathrm{~km} \mathrm{~s}^{-1}$ systematic error. For the bright quasars with many observations, we estimate a typical systematic error due to this scatter of $10-15 \mathrm{~km} \mathrm{~s}^{-1}$.

In addition to the scatter, there are systematic offsets produced by the Sun angle of the telescope and its projected heliocentric motion (possibly an inaccuracy in the star-tracker) that were measured and corrected by de Vries et al. (2015); we refer the reader to their paper for a detailed description. The earliest version of SAS that conained these corrections was v13.0.0, and it was 


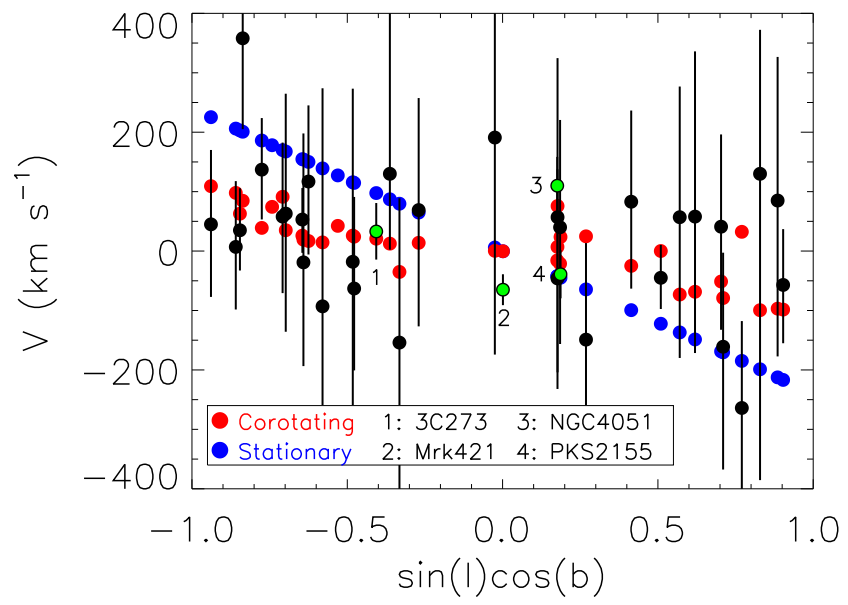

Figure 3. RGS measurements of O VII velocity offsets as a function of $\sin (l) \cos (b)$. Two models are shown for comparison with the data (black points): a stationary model (blue points) and a corotating model (red points). Error bars are $1 \sigma$ (standard deviation). The four objects with the smallest error bars are labeled 1-4. The velocities of the lines towards Mrk 421 and NGC 4051 (labeled '2' and ' 3 ') suggest some intrinsic scatter.

not enabled by default until v14.0.0; all prior absorptionline halo studies using the RGS used an earlier version or did not mention the correction. We measured line centroids with and without these corrections, and found centroid shifts in our sample with magnitudes between $0-100 \mathrm{~km} \mathrm{~s}^{-1}$ (higher for weak lines). To show the effect of not including them, we show the measured centroids without these corrections in Table 1 Finally, if we used the default (proposal) coordinates instead of the SIMBAD coordinates, we measured offsets of $\pm 10 \mathrm{~km} \mathrm{~s}^{-1}$.

Thus, we would expect our measured centroids to be correlated with prior results but perhaps significantly different relative to the statistical errors. For example, there is a systematic offset of about $60 \mathrm{~km} \mathrm{~s}^{-1}$ between our measurements and Fang et al. (2015) for the several systems where it can be measured. This offset cannot be entirely explained by the Sun angle and heliocentric motion corrections, but it is consistent with shifts seen relative to prior versions of SAS (de Vries et al. 2015). They do not report the line centroids for active stars, as they only need to measure the centroids to sufficient accuracy to identify halo absorbers. Thus, we do not regard the apparent inconsistency as reflecting an inherent uncertainty in line centroid measurements.

\subsection{Other Systematics}

In addition to the wavelength grid, there are a few sources of systematic uncertainty and some fitting choices that affect our final results but where we believe there is a correct choice. We briefly describe these here.

Cool Pixels - There are several "cool" pixels in the vicinity of $21.6 \AA$ that have a lower than expected signal (by about $20 \%$ ). By default, these pixels are included in the spectrum. We exclude them because they can affect weak absorption features. The typical velocity shift between keeping and excluding them is $\Delta v=10 \mathrm{~km} \mathrm{~s}^{-1}$ in bright sources.
Binning and Resampling - The native RGS binning is about $11 \mathrm{~m} \AA$ at $21.6 \AA$, but the default processing resamples the events onto a user-specified grid, with a default value of $10 \mathrm{~m} \AA$. This resampling is probabilistic with a random element (normally distributed), which means that running the same protocol on a given data set multiple times will result in slightly different spectra. The magnitude of the velocity shift is strongly dependent on the line strength and the continuum $S / N$, so we reprocessed each data set ten times per reduction protocol set. We then added the standard deviation in the measured velocities in quadrature to the statistical error, since it behaves in essentially the same way. The bin sizes also affect the measured velocities, with a mean line centroid shift of $\Delta v=7 \mathrm{~km} \mathrm{~s}^{-1}$ between bins of $10 \mathrm{~m} \AA$ and $20 \mathrm{~m} \AA$ in bright systems (we use the latter).

Stacking - Temporal changes in the instrumental response or changes in the spectral shape of the source can bias the results of stacked spectra. On the other hand, jointly fitting several low- $S / N$ spectra with the continuum shape as a free parameter leads to poorer constraints on the velocity of a line based on spectral bins far from the absorption line. We stack the spectra to improve the continuum $S / N$, but to determine if this provides systematic bias we measured the line shift between joint fits and stacked spectra in our brightest sources and the calibration sources. We find a typical $\Delta v=5 \mathrm{~km} \mathrm{~s}^{-1}$ because the instrumental response in these regions does not appear to have temporal changes.

Line Profile and Fixed Line Width-For lines with Doppler $b<200 \mathrm{~km} \mathrm{~s}^{-1}$, we do not expect to measure reliable line widths (however, see the Doppler $b$ measurements in some of the same lines in Fang et al. 2015). Since the RGS line-spread function is best described as a Lorentzian near the core, we fit our spectra using a Lorentzian profile with the width fixed at the instrumental line width. If we use a Gaussian line profile instead but keep the width fixed, the line shift is consistent with zero in most cases but can be up to $\Delta v=30 \mathrm{~km} \mathrm{~s}^{-1}$ in weak lines (statistical errors greater than $200 \mathrm{~km} \mathrm{~s}^{-1}$ ). When the line width is a free parameter, we find shifts in the Lorentzian centroids of $\Delta v=0-15 \mathrm{~km} \mathrm{~s}^{-1}$ (the shift magnitude is negatively correlated with $S / N$ ) and $\Delta v=20-50 \mathrm{~km} \mathrm{~s}^{-1}$ in the Gaussian case. However, for the Gaussian lines the best-fit line widths tend to be $1.5-2$ times the instrumental resolution, and the statistical error also increases. These fits are usually not significantly better than with a fixed line width, so in our view a non-zero line width is not required by the data, and these line widths reflect some small curvature in the continuum.

Bandpass - The fitting bandpass is important because the continuum model needs to fit well even if the result is unphysical. Typically, one fits the local continuum, but in the literature for the $\mathrm{O}$ VII line this can vary from a fitting interval less than $1 \AA$ wide to about $5 \AA$ wide. Our choice of fitting bandpass (21-22 $\AA$ ) is motivated by strong instrumental features below $21 \AA ̊$ and above $22 \AA$, but if we ignore these features and expand our bandpass by $\pm 1 \AA$, the typical velocity shift is $\Delta v=3 \mathrm{~km} \mathrm{~s}^{-1}$. 
Table 1

Oxygen Absorption Line Sample

\begin{tabular}{|c|c|c|c|c|c|c|c|c|c|c|}
\hline Name & $\begin{array}{l}\text { Gal Long. } \\
\text { (deg) }\end{array}$ & $\begin{array}{c}\text { Gal Lat. } \\
\text { (deg) }\end{array}$ & $z$ & $\sin (l) \cos (b)$ & $\begin{array}{c}v_{0} \\
\left(\mathrm{~km} \mathrm{~s}^{-1}\right)\end{array}$ & $\begin{array}{c}v_{0}-v_{0, \mathrm{nc}} \\
\left(\mathrm{km} \mathrm{s}^{-1}\right)\end{array}$ & $\begin{array}{c}\text { Corotating } \\
\left(\mathrm{km} \mathrm{s}^{-1}\right)\end{array}$ & $\begin{array}{c}\text { Stationary } \\
\left(\mathrm{km} \mathrm{s}^{-1}\right)\end{array}$ & $\begin{array}{c}\text { Eq. Width } \\
(\mathrm{m} \AA)\end{array}$ & Cont. $S / N$ \\
\hline Mkn 421 & 179.832 & 65.031 & 0.0300 & 0.0013 & $-65_{-21}^{+21}$ & -29 & 0 & 0 & $13.1 \pm 0.5$ & 232 \\
\hline $3 \mathrm{C} 273$ & 289.951 & 64.360 & 0.1580 & -0.4069 & $33_{-45}^{+45}$ & -27 & 21 & 98 & $24.6 \pm 1.6$ & 55 \\
\hline PKS 2155-304 & 17.730 & -52.245 & 0.1160 & 0.1865 & $\begin{array}{l}-39_{-38}^{+38} \\
\end{array}$ & -36 & 24 & -45 & $15.4 \pm 0.9$ & 95 \\
\hline Mkn 509 & 35.971 & -29.855 & 0.0340 & 0.5094 & $-45_{-50}^{+53}$ & -41 & 0 & -122 & $30.2 \pm 2.4$ & 48 \\
\hline NGC 4051 & 148.883 & 70.085 & 0.0023 & 0.1761 & $110_{-41}^{+46}$ & 8 & -16 & -42 & $40.4 \pm 3.6$ & 33 \\
\hline MCG -06-30-15 & 313.292 & 27.680 & 0.0077 & -0.6448 & $53_{-54}^{+51}$ & -1 & 26 & 154 & $35.1 \pm 3.2$ & 38 \\
\hline Ark 564 & 92.138 & -25.337 & 0.0247 & 0.9032 & $-57_{-97}^{+93}$ & 21 & -98 & -217 & $12.3 \pm 1.9$ & 50 \\
\hline ESO 141-55 & 338.183 & -26.711 & 0.0371 & -0.3323 & $-154_{-264}^{+242}$ & 102 & -35 & 80 & $21.2 \pm 5.1$ & 18 \\
\hline $\mathrm{H} 1426+428$ & 77.487 & 64.899 & 0.1290 & 0.4142 & $83_{-145}^{+153}$ & -7 & -25 & -99 & $16.0 \pm 3.9$ & 25 \\
\hline 1H 0707-495 & 260.169 & -17.672 & 0.0405 & -0.9388 & $45_{-121}^{+124}$ & -21 & 109 & 225 & $24.3 \pm 6.1$ & 22 \\
\hline PKS 0558-504 & 257.962 & -28.569 & 0.1370 & -0.8589 & $7_{-104}^{+110}$ & 8 & 98 & 206 & $16.6 \pm 4.2$ & 32 \\
\hline NGC 4593 & 297.483 & 57.403 & 0.0090 & -0.4781 & $-63_{-137}^{+153}$ & 11 & 24 & 115 & $27.1 \pm 7.1$ & 13 \\
\hline Mrk 335 & 108.763 & -41.424 & 0.0258 & 0.7100 & $\begin{array}{l}-161_{-206}^{+158} \\
+15\end{array}$ & -40 & -79 & -170 & $19.3 \pm 5.3$ & 22 \\
\hline PG $1211+143$ & 267.552 & 74.315 & 0.0809 & -0.2702 & $69_{-195}^{+188}$ & -57 & 14 & 65 & $40.3 \pm 11$ & 7 \\
\hline PG $1244+026$ & 300.041 & 65.214 & 0.0482 & -0.3631 & $130_{-127}^{+286}$ & -188 & 12 & 87 & $40.8 \pm 11$ & 10 \\
\hline Mkn 501 & 64.600 & 38.860 & 0.0337 & 0.7034 & $41_{-173}^{+155}$ & -68 & -51 & -169 & $25.2 \pm 10$ & 22 \\
\hline $1 \mathrm{ES} 1028+511$ & 161.439 & 54.439 & 0.3600 & 0.1853 & $40_{-196}^{+180}$ & 81 & -21 & -44 & $36.4 \pm 11$ & 12 \\
\hline ESO 198-24 & 271.639 & -57.948 & 0.0455 & -0.5305 & $-652_{-219}^{+231}$ & -393 & 42 & 127 & $62.2 \pm 19$ & 7 \\
\hline NGC 2617 & 229.300 & 20.939 & 0.0142 & -0.7079 & $58_{-128}^{+124}$ & -95 & 91 & 170 & $30.1 \pm 9.5$ & 10 \\
\hline PG $1553+113$ & 21.909 & 43.964 & 0.3600 & 0.2686 & $-149_{-175}^{+162}$ & 45 & 25 & -64 & $27.6 \pm 8.7$ & 13 \\
\hline H1101-232 & 273.190 & 33.079 & 0.1860 & -0.8367 & $358_{-152}^{+141}$ & 2 & 85 & 201 & $45.5 \pm 17$ & 8 \\
\hline 3C 390.3 & 111.438 & 27.074 & 0.0561 & 0.8289 & $130_{-515}^{+242}$ & -21 & -99 & -199 & $25.0 \pm 10$ & 10 \\
\hline 1H 0419-577 & 266.963 & -42.006 & 0.1040 & -0.7421 & $783_{-255}^{+273}$ & 17 & 74 & 178 & $39.9 \pm 16$ & 6 \\
\hline Fairall 9 & 295.073 & -57.826 & 0.0470 & -0.4825 & $-18_{-333}^{+291}$ & -226 & 26 & 116 & $37.2 \pm 15$ & 6 \\
\hline IRAS 13224-3809 & 310.189 & 23.979 & 0.0660 & -0.6982 & $63_{-198}^{+201}$ & 31 & 35 & 167 & $45.1 \pm 19$ & 7 \\
\hline NGC 5408 X-1 & 317.149 & 19.496 & 0.0017 & -0.6414 & $-19_{-174}^{+216}$ & -98 & 19 & 154 & $30.4 \pm 14$ & 7 \\
\hline PDS 456 & 10.392 & 11.164 & 0.1840 & 0.1770 & $57_{-261}^{+267}$ & 47 & 76 & -42 & $60.0 \pm 29$ & 7 \\
\hline Mrk 279 & 115.042 & 46.865 & 0.0305 & 0.6195 & $58_{-229}^{+278}$ & -29 & -68 & -149 & $14.2 \pm 7.0$ & 15 \\
\hline $\mathrm{E} 1821+643$ & 94.003 & 27.417 & 0.2970 & 0.8855 & $85_{-262}^{+241}$ & -28 & -97 & -212 & $35.7 \pm 18$ & 7 \\
\hline IC $4329 \mathrm{~A}$ & 317.496 & 30.920 & 0.0160 & -0.5799 & $-93_{-229}^{+367}$ & -73 & 15 & 139 & $38.3 \pm 20$ & 7 \\
\hline PG $0804+761$ & 138.279 & 31.033 & 0.1000 & 0.5704 & $57_{-236}^{+219}$ & 98 & -73 & -137 & $28.5 \pm 15$ & 6 \\
\hline NGC 5548 & 31.960 & 70.496 & 0.0172 & 0.1768 & $-46_{-185}^{+183}$ & -51 & 7 & -42 & $18.0 \pm 10$ & 13 \\
\hline Mrk 766 & 190.681 & 82.270 & 0.0129 & -0.0249 & $191_{-365}^{+240}$ & 93 & 0 & 6 & $6.0 \pm 4.2$ & 27 \\
\hline \multicolumn{11}{|c|}{ Local Group and Halo Sources } \\
\hline LMC X-3 & 273.576 & -32.082 & - & -0.8457 & $35_{-66}^{+69}$ & 7 & 63 & 203 & $23.1 \pm 3.0$ & 36 \\
\hline $4 \mathrm{U} 1957+11$ & 51.308 & -9.330 & - & 0.7702 & $-264_{-152}^{+145}$ & -124 & 32 & -185 & $20.6 \pm 4.1$ & 22 \\
\hline MAXI J0556-032 & 238.939 & -25.183 & - & -0.7751 & $137_{-82}^{+85}$ & 84 & 39 & 186 & $16.0 \pm 3.3$ & 33 \\
\hline SMC X-1 & 300.414 & -43.560 & - & -0.6251 & $117_{-122}^{+127}$ & -3 & 17 & 150 & $21.0 \pm 4.9$ & 13 \\
\hline
\end{tabular}

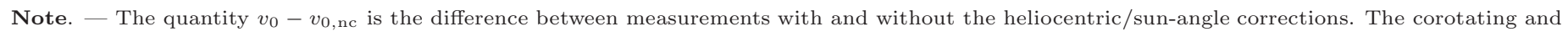

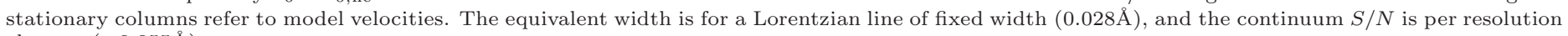
element ( $\sim 0.055 \AA)$.

Bad Columns near 21.6 - There is asymmetry in the line-spread function (LSF), which is the instrumental response to a $\delta$-function, near the $\mathrm{O}$ VII line. This is not the same as the well known asymmetry in the LSF at the $1 \%$ level in the line wings (which is not important), but rather due to bad columns that are not included in the cool pixel list (Figure 2). For an arbitrarily strong $\delta$ function line, the offset in the measured centroid from this defect can range from $\Delta v=0-100 \mathrm{kms}^{-1}$ depending on where the true line centroid is. However, if the line is unresolved but has some physical width (so that incident photons would be dispersed over multiple bins even before the LSF spreads them out), the error is strongly mitigated. We used XSPEC simulations to determine the error as a function of Doppler $b$ parameter, and for $b>40 \mathrm{kms}^{-1}$ (about the thermal width even without turbulence), the typical error is reduced to $15 \mathrm{~km} \mathrm{~s}^{-1}$ (Figure 2). We expect the Galactic halo lines to be in this regime. Alternatively, one can ignore the columns, but because of their proximity to the region of interest, this will also bias the results.

Overall, the systematic error should allow measurements to better than $30 \mathrm{~km} \mathrm{~s}^{-1}$ accuracy with sufficient $S / N$. For the high $S / N$ lines we estimate a typical systematic error of $15-20 \mathrm{~km} \mathrm{~s}^{-1}$, and for lines weaker than about $4 \sigma$, the systematic error is dominated by the sta- 

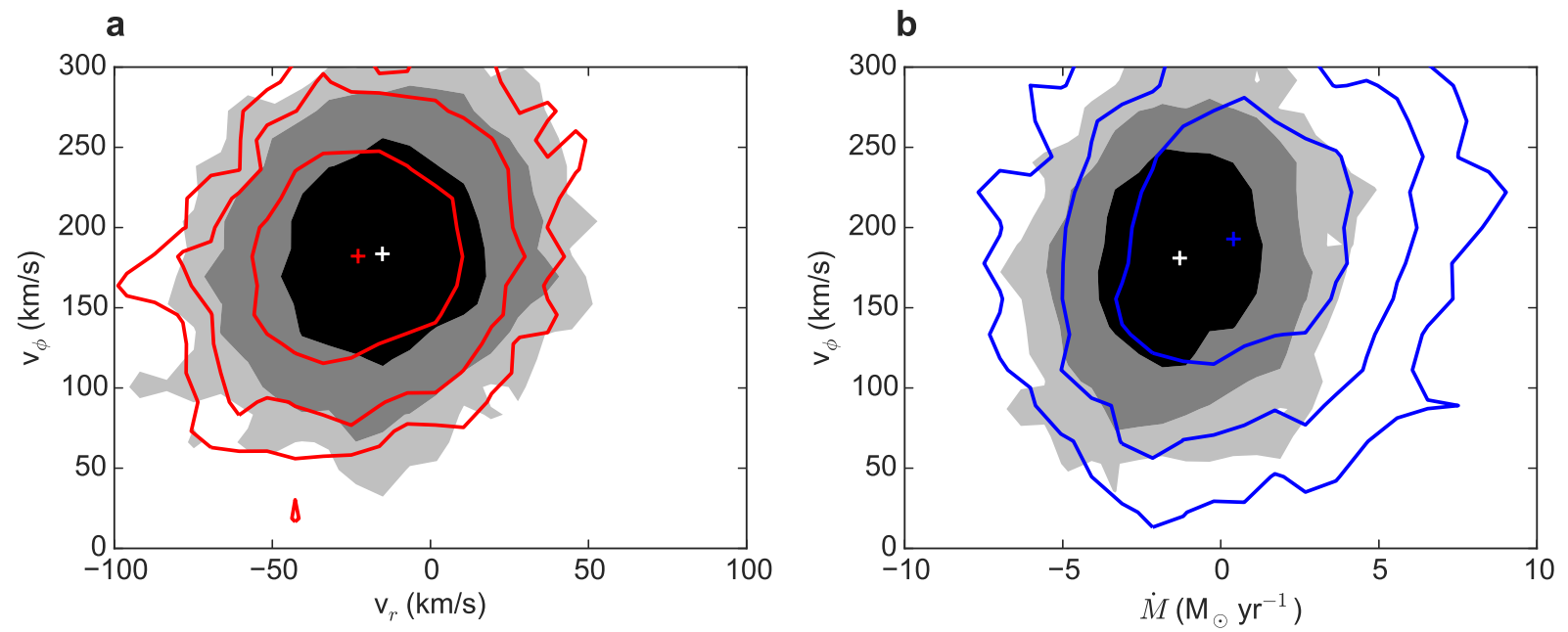

C
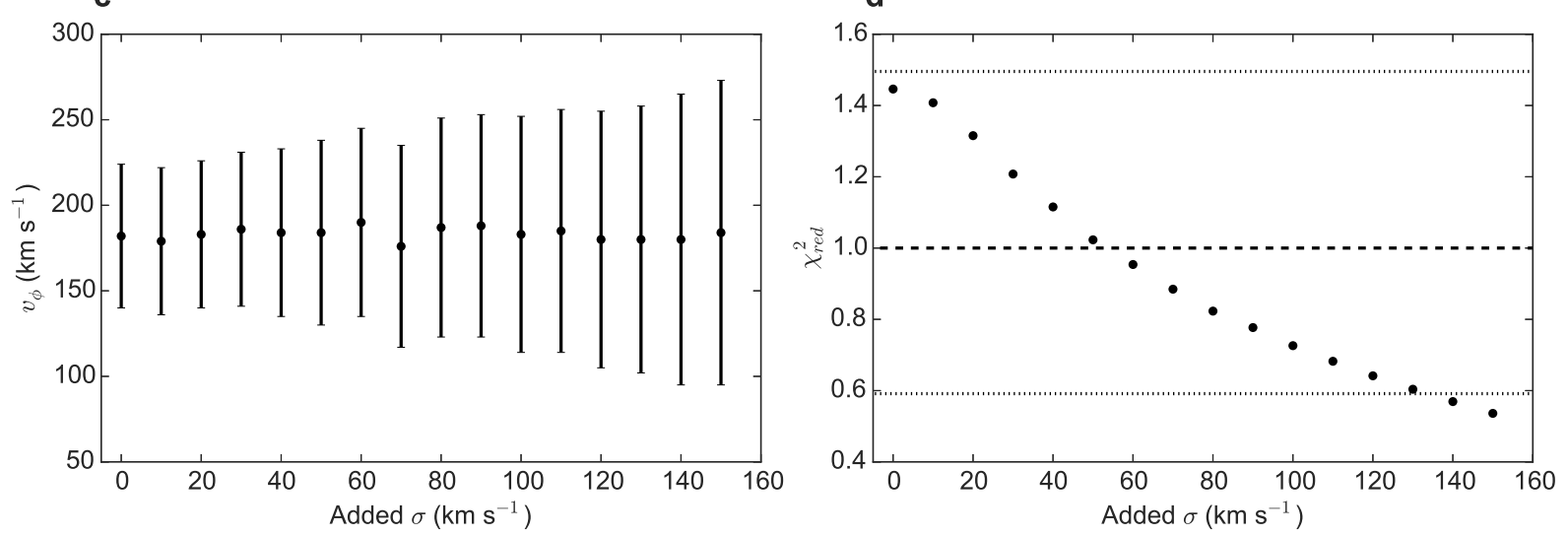

Figure 4. (a) Contours show the 1,2, and $3 \sigma$ deviations from the best-fit parameters for the whole sample (shaded) and lines with measurement errors less than $250 \mathrm{~km} \mathrm{~s}^{-1}$ (lines). (b) The radial velocity can be converted to an accretion or outflow rate, which is consistent with zero. (c) When the possibility of intrinsic scatter is included in the model, $v_{\phi}$ becomes less certain, but even with $150 \mathrm{~km} \mathrm{~s}^{-1}$ of added scatter, some rotation is necessary. (d) The added scatter value that brings the reduced $\chi^{2}$ closest to 1.0 is near $50 \mathrm{~km} \mathrm{~s}{ }^{-1}$, with values above $140 \mathrm{~km} \mathrm{~s}^{-1}$ ruled out with $90 \%$ confidence (dotted lines).

tistical error.

\section{HALO MODEL}

We used a simple halo model for comparison to the data. We adopted an extended density profile (Miller \& Bregman 2015) in which

$$
n(r)=n_{0}\left(1+\left(r / r_{c}\right)^{2}\right)^{-3 \beta / 2} \mathrm{~cm}^{-3}
$$

where $n_{0} r_{c}^{3 \beta}=1.35 \pm 0.24 \mathrm{~cm}^{-3} \mathrm{kpc}^{3 \beta}$ and $\beta=0.50 \pm$ 0.03 , computed on a grid with $0.05 \mathrm{kpc}$ cells. This model describes a large, all-sky sample of 649 O VII and OvIII emission lines (Henley et al. 2010; Henley \& Shelton $2012)$ well. We then imposed global bulk radial $\left(v_{r}\right)$ or azimuthal $\left(v_{\phi}\right)$ velocities, assuming that these are constant with radius. These are the free parameters in the model. We likewise assume a constant metallicity $\left(Z=0.3 Z_{\odot}\right)$ and a Doppler $b$ parameter of $85 \mathrm{~km} \mathrm{~s}^{-1}$ due to random turbulent motion in each cell, based on hydrodynamic simulations (Cen 2012). We also account for the possibility of intrinsic scatter (resulting from hydrodynamic flows) about any model by adding a velocity dispersion to the velocities when comparing to the models. This dispersion is not the same as line broadening, but refers to the typical value for a distribution of cen- troid shifts.

We obtain model velocities for each object by integrating from the position of the Sun outward along each line of sight, computing the line-of-sight velocity component and broadening for each cell and summing the resultant Voigt profiles Miller et al. (a more detailed description is given in 2016). We convolve the result with the LSF, compute the centroid, and add the solar reflex motion for comparison to the observations. In other words, the velocities are compared in the frame of the Local Standard of Rest. For this model, at least $50 \%$ of the total absorption comes from beyond $7 \mathrm{kpc}$ from the Sun, and $90 \%$ from within $50 \mathrm{kpc}$. In the simplest case of a stationary halo, the Galactic rotation of the Local Standard of Rest (Reid et al. 2014) $\left(v_{\mathrm{LSR}}=240 \pm 8 \mathrm{~km} \mathrm{~s}^{-1}\right)$ is reflected in the measured Doppler shifts: $v_{\text {obs }}=-240 \sin (l) \cos (b)$, where $l$ is the Galactic longitude and $b$ the Galactic latitude. The product $\sin (l) \cos (b)= \pm 1$ corresponds to the Sun moving directly towards or away from that direction, resulting in a Doppler shift of $\mp 240 \mathrm{~km} \mathrm{~s}^{-1}$. Another simple case is a corotating halo $\left(v_{\phi}=v_{\mathrm{LSR}}\right)$, in which case the Doppler shifts will be closer to zero. Figure 3 shows the measured velocities with the stationary and corotating models. 

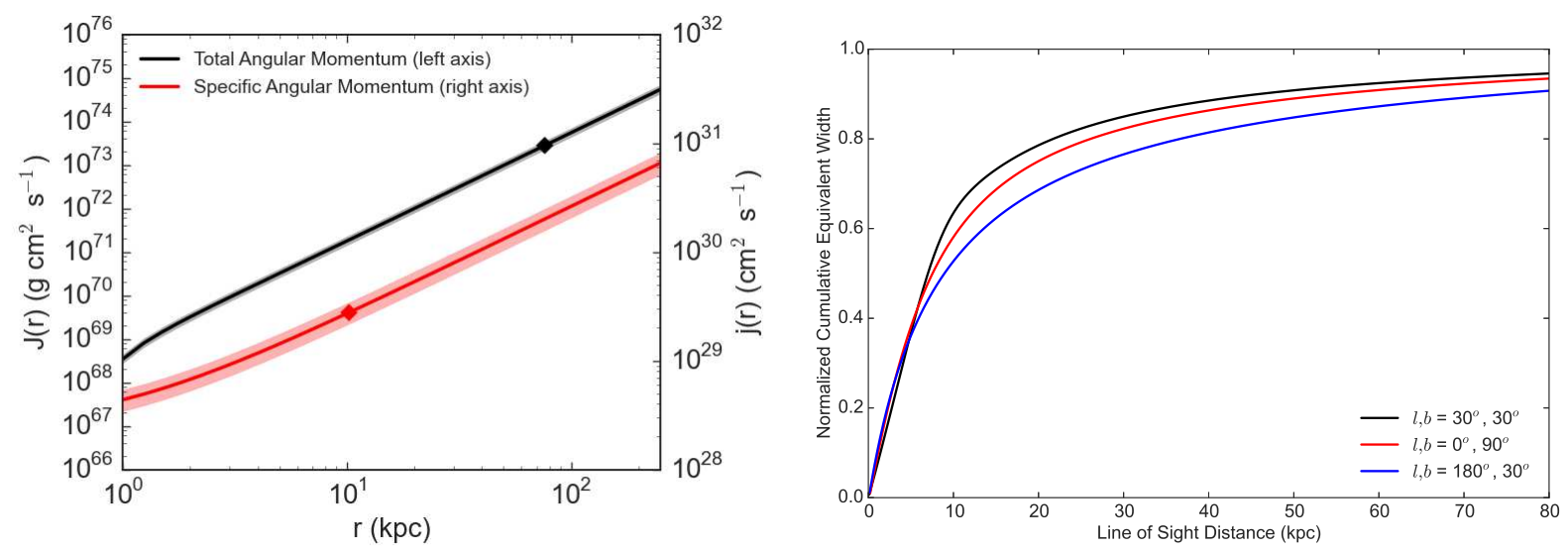

Figure 5. (a) The black (red) line shows the total (specific) angular momentum of the hot gas as a function of radius in our density model. Both assume $v_{\phi}=183 \pm 41 \mathrm{~km} \mathrm{~s}^{-1}$. The diamonds show the values for the sum of stellar and Hi values in the disk of the Milky Way. (b) The cumulative equivalent width for three sightlines shows that, while different sightlines are sensitive to gas at different distances, in all cases more than $70 \%$ of the equivalent width comes from beyond $5 \mathrm{kpc}$ from the Sun.

\section{RESULTS}

The best-fit $v_{r}$ and $v_{\phi}$ values were obtained from a Markov-chain Monte Carlo (MCMC) approach, using the $\chi^{2}$ statistic as a goodness-of-fit parameter. For zero dispersion, the best model has a prograde rotation velocity of $v_{\phi}=183 \pm 41 \mathrm{~km} \mathrm{~s}^{-1}$ and a global inflow of $v_{r}=-15 \pm 20 \mathrm{~km} \mathrm{~s}^{-1}$, corresponding to a net accretion rate of $\dot{M}=1 \pm 2 M_{\odot} \mathrm{yr}^{-1}$ (Figure (4). This is a formally acceptable fit, whereas the stationary halo is rejected with $99.95 \%$ probability and the corotating halo is marginally rejected with $95 \%$ probability. The velocity dispersion for which the reduced $\chi^{2}$ is closest to 1.0 is about $50 \mathrm{~km} \mathrm{~s}^{-1}$ (Figure 4); substantially more than this produces a $\chi^{2}$ that is too small for the observed line centroids. The apparent inflow is not statistically significant, and the suggestion of inflow primarily results from Mrk 421 (Figure 3), which has a small uncertainty and is near $\sin (l) \cos (b)=0$. Taking these results at face value, the large $v_{\phi}$ and extent of the halo imply that the total angular momentum of the hot gas within $50 \mathrm{kpc}$ is comparable to that in the stars and gas in the disk of the Galaxy (Mo et al. 2010) (Figure 5). The spread in recent measurements of $v_{\mathrm{LSR}}=200-250 \mathrm{~km} \mathrm{~s}^{-1}$ (Brunthaler et al. 2011; Bovy et al. 2009; Reid et al. 2014) leads to $v_{\phi}=$ $130-180 \mathrm{~km} \mathrm{~s}^{-1}$, which does not change the picture of a lagging halo with prograde rotation.

In addition to parameter fitting, we used nonparametric statistical tests to test the hypothesis that some rotation is necessary. We tested the stationary and corotating models using the sign test and the KolmogorovSmirnov (KS) test, which compare distributions and are less sensitive to scatter. The sign test asks whether the medians of the measured and model velocities are consistent with each other (assuming a binomial distribution and a $50 \%$ probability that the model velocity exceeds the measured velocity). The left panel of Figure 6 shows the residuals from subtracting the data from the stationary model values, and the strong asymmetry rules out the stationary model with $99.87 \%$ probability. The KS test compares the cumulative distributions of the measured and model velocities, and this test rules out the stationary halo with $98.5 \%$ probability. The corotating model is acceptable in the sign test (43\% rejection probability) and not in the KS test (99.8\% probability). The rejection of the stationary halo is model independent. If we exclude weak lines with $\sigma_{\text {stat }} \geq 250 \mathrm{~km} \mathrm{~s}^{-1}$, a stationary model is still ruled out at more than $99.4 \%$ probability. If we exclude ambiguous lines (such as those towards NGC 5408 X-1, NGC 4051, and MCG-6-30-15), a stationary model is ruled out at about $98 \%$ probability in the KS test and $99.6 \%$ in the sign test. Excluding NGC 4051 (with its small error bars) increases the range of acceptable $v_{\phi}$ in the parametric fits, but the best-fit $v_{r}$ does not change much as the error bars for Mrk 421 are even smaller.

We investigated the sensitivity of the best-fit parameters and the implied angular momentum to the model assumptions. First, the assumption of constant velocity must break down at some radius. An effort to measure the Galactic rotation curve to $200 \mathrm{kpc}$ using disk and non-disk objects found (Bhattachariee et al. 2014) that $v_{\phi}$ is flat to $80 \mathrm{kpc}$, while a measurement from disk stars in the Sloan Digital Sky Survey found (Xue et al. 2008) that $v_{\phi}$ in the disk declines by $15 \%$ from $v_{\mathrm{LSR}}$ at $10-20 \mathrm{kpc}$ and remains constant from $20-55 \mathrm{kpc}$ (the maximum probed). Thus, the assumption of a constant $v_{r}$ or $v_{\phi}$ within $r<80 \mathrm{kpc}$ appears to be reasonable, and more than $80 \%$ of the cumulative equivalent width comes from within this region (Figure 5). When we considered a model with a constant $v_{\phi}$ within $50 \mathrm{kpc}$ and $v_{\phi}=0 \mathrm{~km} \mathrm{~s}^{-1}$ beyond, the best-fit $v_{\phi}$ is nearly identical to the reported value. For a $10 \mathrm{kpc}$ cutoff, the best-fit $v_{\phi}$ increases.

Second, there may be a metallicity gradient in the hot gas, in which case the cumulative equivalent width will be even more dominated by nearby gas. This would impact the inferred mass and total angular momentum of the halo gas. Constraints from OviI and OviII and pulsar dispersion measures towards the Large Magellanic Cloud are consistent (Miller \& Bregman 2015) with a gradient of $Z(r) \propto r^{-0.2}$ with $Z=0.39 Z$. at $1 \mathrm{kpc}$ and $Z=0.26 Z_{\odot}$ at $10 \mathrm{kpc}$. The uncertainty is large, but the gradient is shallow enough that gas beyond a few kpc contributes $50 \%$ or more of the equivalent width. 

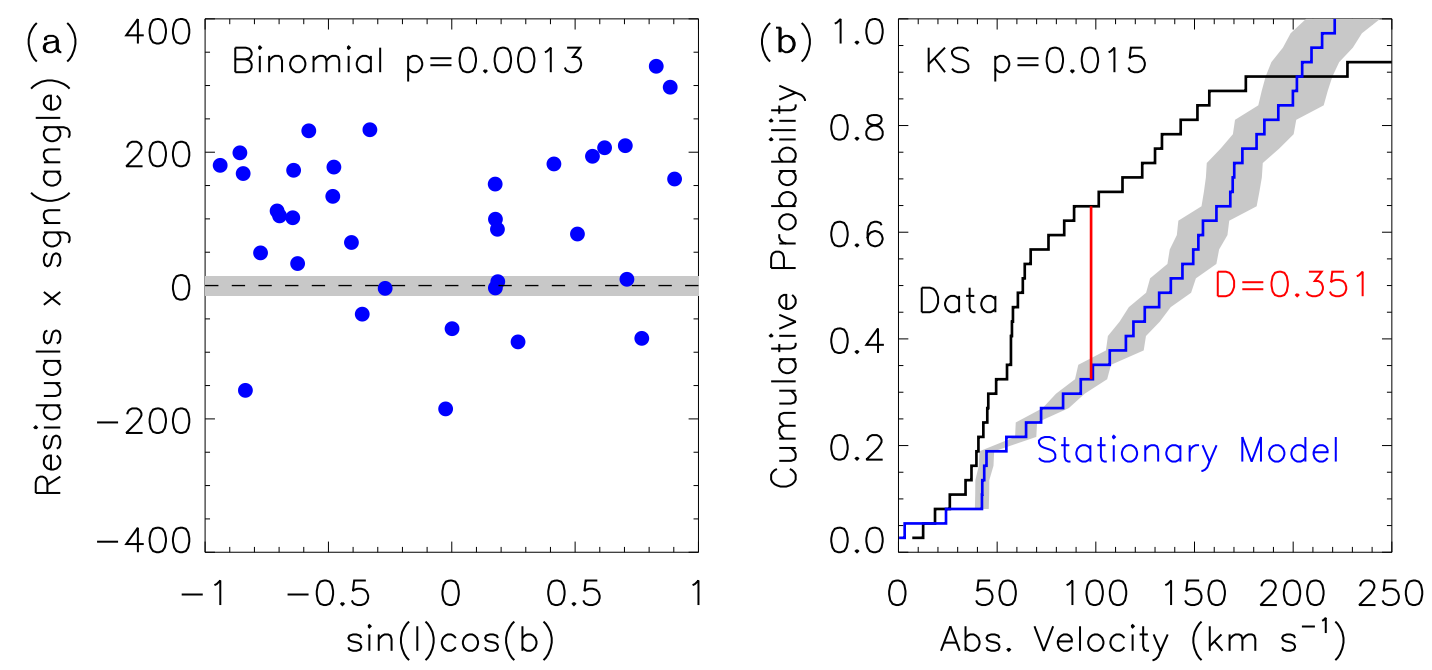

Figure 6. (a). The residual distribution around the stationary model shows that too many sight lines have velocities less than the model predictions. At each angle, a positive residual indicates that the model has a higher velocity magnitude than the measured offset. (b). The cumulative distributions of the model and measured velocity magnitudes (right panel) also rule out a stationary model by the KS test at the $98 \%$ level. The shaded grey regions in each panel represent $\pm 20 \mathrm{~km} \mathrm{~s}^{-1}$ from $v_{\mathrm{LSR}}=240 \mathrm{~km} \mathrm{~s}$.

Finally, even a single-component hot halo is probably not isothermal and may also have flows arising from satellite galaxy motions or gaseous inflows and outflows. Since the combined emission- and absorption-line data favor an extended halo and its temperature is near the virial temperature, the gas is probably volume filling. Hydrodynamic flows in such a halo will primarily induce scatter of the type described above, which does not strongly affect our $v_{\phi}$ measurement (Figure 44). Thus, the measured halo rotation probably extends to tens of kpc and possibly to $100 \mathrm{kpc}$, and this gas will have a substantial angular momentum. However, the angular momentum values (Figure 5) are strongly model dependent, especially due to the assumption of constant $v_{\phi}(r)$.

\section{DISCUSSION}

\subsection{Halo and Disk Models}

The interpretation of the measured Doppler shifts as a rotation signature depends on the validity of our singlecomponent halo model. This depends on the following assumptions: (1) A volume-filling spherical halo is an approximately accurate representation of most of the hot gas around the Galaxy; (2) there is no strong local absorber that we have ignored; (3) The spherical halo has bulk global motion; (4) the assumption of $d v_{\phi} / d R=0$ is reasonable within about $50 \mathrm{kpc}$; and (5) the Doppler shift measurements are accurate. The fourth and fifth assumptions were addressed above, so here we focus on the first three.

Two basic models are suggested in the literature for the structure of the hot halo: an extended, spherical distribution or an exponential disk with a scale height of a few kpc. When using individual sight lines or small samples (Yao et al. 2009a; Hagihara et al. 2010), the observed O VII and O VIII absorption and emission line strengths are consistent with an exponential disk model with a scale height of a few kpc. They are also consistent with a spherical model. However, larger samples of absorption lines ( $\sim 40$ sight lines; Bregman \& Lloyd-Davies 2007; Gupta et al.
2012; Fang et al. 2015) and the all-sky emission-line intensities $(\approx 1,000$ sight lines Henley \& Shelton 2012; Miller \& Bregman 2013, 2015) favor a spherical model. Similar analyses on independent observables that also probe the hot gas, such as the pulsar dispersion measure towards the Large Magellanic Cloud and the rampressure stripping of Milky Way satellites favor an extended halo (Fang et al. 2013; (Salem et al. 2015). Finally, a recent analysis of both Galactic and extragalactic sightlines for $L_{*}$ galaxies finds that the O VII traces hot gas (Faerman et al. 2016). Thus, we adopted the spherical density profile of Miller \& Bregman (2015).

However, these analyses are based on single-component models, and from basic galaxy models we expect at least two X-ray absorbing components: infalling gas that is shock heated to the virial temperature $(T \approx$ $2 \times 10^{6} \mathrm{~K}$ for the Milky Way) and forms an extended halo (e.g., White \& Frenk 1991), and supernova-driven outflows from the disk (e.g., Shapiro \& Field 1976; Hill et al. 2012). In the latter case, we expect an exponential disk of hot gas with a scale height set (for a given Galactocentric radius) by the temperature at the midplane. It is worth noting that for $T=2 \times 10^{6} \mathrm{~K}$, the scale height at the Solar circle is larger than the Galactocentric distance, so the distribution of outflowing gas could also be spherical, if not very extended; the true shape depends on how widespread the outflows are in the disk, the actual midplane temperature, and the amount of radiative cooling. Within the galaxy disk itself, supernovae contribute to the hot interstellar medium, much of which is confined within the disk. Since X-ray absorption covers a wider temperature range than emission, we are also weakly sensitive to cooler gas (described in the following subsection).

These components may be kinematically distinct, as is seen in the warm gas by Nicastro et al. (2003); Savage et al. (2003), but at the relatively low resolution of the RGS, we expect them to be blended. This complicates the interpretation of the measured centroids, which are the weighted average of the offsets for each compo- 
nent along that line of sight. At a qualitative level, we expect the gas confined in the galaxy disk to rotate with it (although depending on the distances to the absorbers, there may not be any rotation signature) and the gas in supernova-driven outflows to rotate in the same direction but lagging the disk as it reaches larger heights or radii.

To constrain the column and thus the influence on the line centroids from a disk component, we extended the analysis of Miller \& Bregman (2015) to fit a disk+halo model to the same data set they used: 648 O VIII emission lines from Henley \& Shelton (2012), which are filtered for contamination from solar-wind charge exchange and ignore most of the Galactic plane. We refer the reader to Miller \& Bregman (2015) for a more detailed explanation of the modeling procedure, but we summarize our model components here.

The Sun exists in a region known as the Local Bubble, which emits soft X-rays (e.g., Cox \& Revnolds 1987; Snowden et al. 1997), and we adopt for the Bubble a temperature of $1.2 \times 10^{6} \mathrm{~K}$, a variable path length between $100-300 \mathrm{pc}$, and a density $n_{\mathrm{LB}}=4 \times$ $10^{-3} \mathrm{~cm}^{-3}$. The nature of the Bubble and the density remain debatable because of the contribution from solar-wind charge exchange (e.g., Welsh \& Shelton 2009: Kuntz 2009; Galeazzi et al. 2014; Snowden et al. 2015; Henley \& Shelton 2015). We have ignored hot interstellar gas confined within the galaxy disk other than the Local Bubble. Constraints from the intensity of the soft Xray background imply that our sight lines will incur only a small column from this material, and the emission-line sample excludes the Galactic plane. For the extended halo component, we assume an isothermal $\left(2 \times 10^{6} \mathrm{~K}\right)$ plasma with a density profile described by Equation 1 For the exponential disk component, we assume a form for the density of

$$
n=n_{0, \mathrm{disk}} e^{-z / h} \mathrm{~cm}^{-3}
$$

where $h$ is the scale height. The temperature declines in an analogous way, and we fix the midplane temperature and its scale height to $2.5 \times 10^{6} \mathrm{~K}$ and $5.6 \mathrm{kpc}$, respectively (Hagihara et al. 2010) because we do not model the line ratios as a temperature diagnostic. The free parameters are the normalizations, $\beta$, and $h$, which we constrain using the same MCMC method as Miller \& Bregman (2015).

We assume that each component is optically thin and compute its contribution to the intensity along each lineof-sight $s$ :

$$
I=\frac{1}{4 \pi} \int n(s)^{2} \epsilon(T(s)) d s
$$

where $\epsilon(T)$ is the volumetric line emissivity from the APEC thermal plasma code (Foster et al. 2012). Finally, the line intensities from Henlev \& Shelton (2012) do not account for absorption due to Galactic HI, so to compare our model intensities to theirs we apply photoelectric absorption using the neutral hydrogen column from Kalberla et al. (2007) to the halo and disk components:

$$
I(s)=I_{\mathrm{LB}}(s)+\left(I_{\text {halo }}(s)+I_{\text {disk }}(s)\right) e^{-\sigma N_{\mathrm{H}}}
$$

where $\sigma$ is the absorption cross-section and $I_{\mathrm{LB}}$ is the intensity from the Local Bubble.
Figure 7 shows the results from the MCMC analysis as marginalized posterior probability distributions for each of the free parameters. The model is not a significant improvement on the pure halo model, and the best-fit parameters for the halo $\left(n_{0, \text { halo }} r_{c}^{3 \beta}=1.43 \pm\right.$ $\left.0.25 \times 10^{-2} \mathrm{~cm}^{-3} \mathrm{kpc}^{3 \beta}, \beta=0.52 \pm 0.03\right)$ are consistent with the results in Miller \& Bregman (2015). The disk parameters are poorly constrained and indicate that the exponential disk contributes at most $10 \%$ of the total $O$ VII column density. This corroborates the Miller \& Bregman (2015) result. In contrast, the Hagihara et al. (2010) disk model (which assumes the halo gas can be described entirely by a disk and is based on one sightline) finds $n_{0 \text {.disk }}=1.4 \times 10^{-3}$ and $h=2.3 \mathrm{kpc}$. Yao \& Wang (2005), who include some sight lines near the Galactic plane and some outside the plane, find that the $\mathrm{O}$ VII column is consistent with $n_{0, \text { disk }}=6.4 \times 10^{-3} \mathrm{~cm}^{-3}$ and a scale height of $h=1.2 \mathrm{kpc}$, but the authors acknowledge that mixing results from sight lines towards nearby X-ray binaries at low latitudes and those at high latitudes can lead to a strong bias; the hot material confined to the galaxy disk is not part of the exponential disk structure that we have modeled, but it can contribute at low latitudes.

Thus, the impact of the disk component on the measured centroids must be small, since $80-90 \%$ of the column density will come from the spherical component. Assuming that the two components are kinematically distinct, for the latter to be stationary and consistent with the measured centroids requires a disk speed much faster than co-rotation. We verified this by modeling a rotating disk and a stationary halo where the disk contributes $10 \%$ of the column. Since this is inconsistent with models where the gas originates in the galaxy disk, the data probe motion in the spherical component. For reference, if the Hagihara et al. (2010) disk model is adopted instead of the Miller \& Bregman (2015) model or our disk +halo model, the best-fit azimuthal and radial velocities are $v_{\phi}=151 \pm 32 \mathrm{~km} \mathrm{~s}^{-1}$ and $v_{r}=-15 \pm 18 \mathrm{~km} \mathrm{~s}^{-1}$.

We have assumed that, to first order, the spherical component moves as a solid body with some $v_{\phi}$ and $v_{r}$. There may be second-order effects such as the internal flows mentioned above (perhaps due to satellite motions or infalling clouds) or modes in the fluid, which would add scatter to the velocity measurements. However, if the halo is volume filling and in steady state, then large scale disturbances will tend to dissipate in a soundcrossing time (which could be a long time if the halo is very extended), and the gas (if stationary) will tend towards hydrostatic equilibrium. Multiple major kinematic components are therefore not expected, although we would expect differential rotation. In this case, the measured rotation velocity is some average but still provides useful information. Also, if the material is fresh infall from the cosmic web then it likely accretes along filaments, which lead to a preferential orbital angular momentum axis. Finally, gas ejected from the disk (either cold or hot) could spin up the halo (Marinacci et al. 2011), although perhaps not to the velocity that we infer.

It is possible that there is a layer of warm-hot gas near the Galactic plane (in addition to the Local Bubble) that affects every sight line out of the Galaxy, but to which our models are not sensitive because we ignore data close 

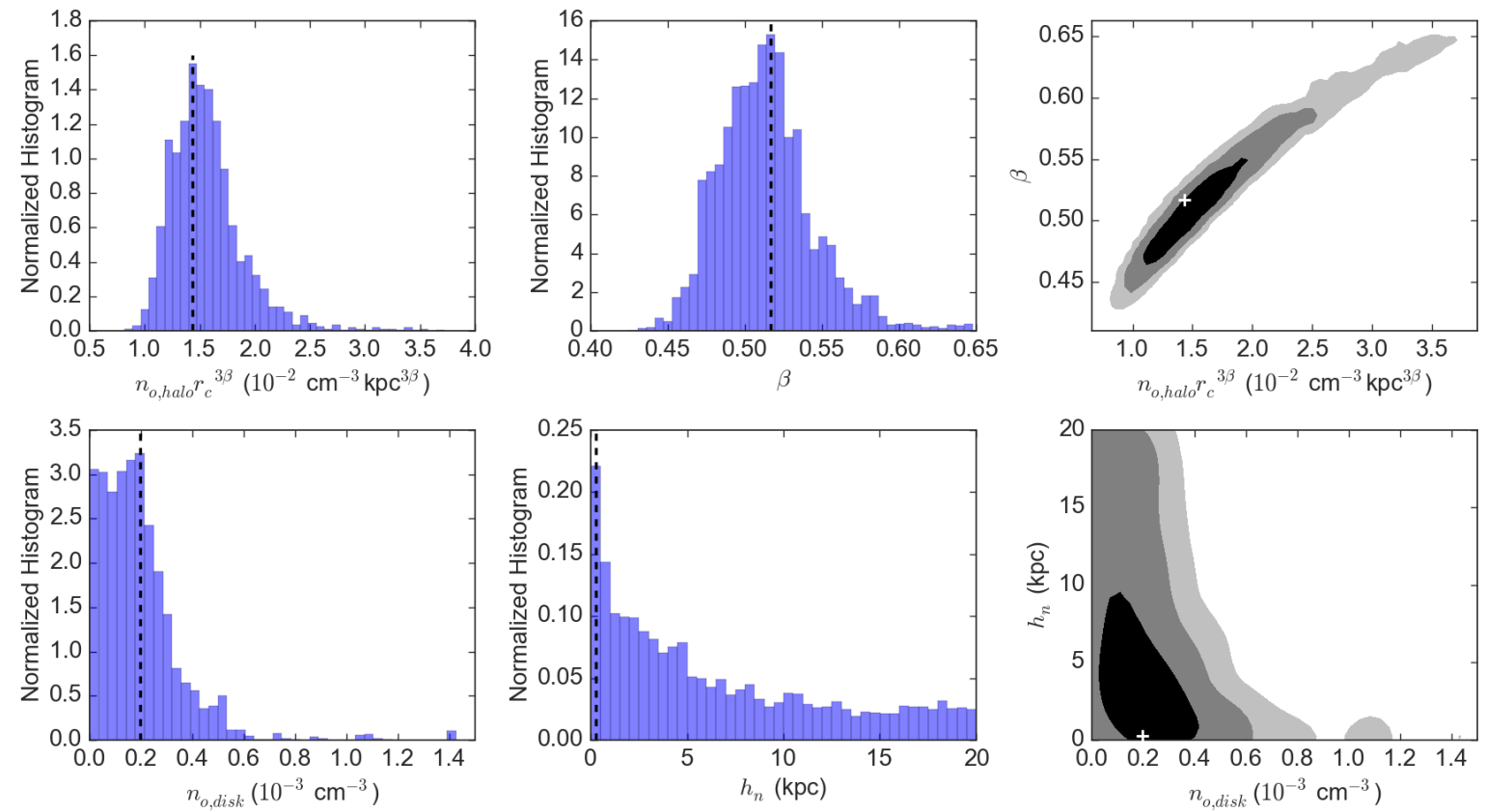

Figure 7. Our MCMC fitting results as marginalized posterior probability distributions (left and center columns) and contour plots for the model parameters (right column). The vertical dashed lines (distribution plots) and white crosses (contour plots) represent the best-fit model values. The halo parameter distributions (top left and top center panels) are almost identical to the results from Miller \& Bregman (2015). The disk midplane density distribution (bottom left panel) implies densities significantly lower than previous estimates $\left(\approx 10^{-3}\right.$ $\left.\mathrm{cm}^{-3}\right)$.

to the Galactic plane (where dust, supernova remnants, and other features make modeling very difficult). Characterizing the structure and filling factor of the hot interstellar medium has been a major effort by itself (e.g., Yao \& Wang 2005; Nicastro et al. 2016; Hagihara et al. 2011) and is beyond the scope of this paper, but if there is such a layer (possibly a disk with a scale height of a few hundred pc) with a high density, the rotation signature in the RGS data could be misattributed to the halo. On the other hand, for plausible path lengths, oxygen column densities, and foreground absorption column densities this layer should also produce emission in excess of the Local Bubble contribution to the soft X-ray background.

To summarize, the halo models that are based on many data points as opposed to a few sightlines favor a spherical halo (especially in emission) as the dominant component. Any contribution to the column density from a kinematically distinct (thick) exponential disk is small in this scenario, so the Doppler shifts support a nonstationary extended halo. Reinterpreting these shifts will be necessary if future data or analyses rule out a spherical halo (or at least a volume-filling one) or a high resolution $\mathrm{X}$-ray spectrometer resolves the lines into components inconsistent with rotation. Also, we reiterate that the parametric fit strongly depends on the few AGNs with the highest $S / N$, but the nonparametric tests indicate rotation at some level.

\subsection{Potential Bias from Cooler Gas}

The O VII ion fraction is high between $3-20 \times 10^{5} \mathrm{~K}$, so the cooler (non-coronal) gas seen in and around the Galaxy in O VI with FUSE (Nicastro et al. 2003;
Savage et al. 2003; Wakker et al.|2003; Wang et al.|2005; Yao \& Wang 2007; Shelton et al. 2007; Bowen et al. 2008) will also absorb O VII. The O VI lines towards background objects reveal Galactic and highvelocity absorbers (Nicastro et al. 2003; Savage et al. 2003; Sembach et al. 2003). The Galactic absorbers (also seen towards stars) are consistent with an exponential disk of scale height $1-4 \mathrm{kpc}$ (Savage et al. 2003; Bowen et al. 2008), whereas the high-velocity absorbers may come from the halo or outside the Galaxy. If the column densities of these O VI absorbers are high, then their O VII lines could bias our results. Since the disk component will rotate, we are most concerned about contamination from this gas.

Bowen et al. (2008) measured O VI column densities for about 150 sight lines around the Galaxy and found a typical column of $N_{\text {OVI }} \sin (b) \sim 1.6 \times 10^{14} \mathrm{~cm}^{-2}$. For the sight lines in our sample, we expect $N_{\text {OVI }}=1-4 \times$ $10^{14} \mathrm{~cm}^{-2}$. If the $\mathrm{O}$ VI absorbers are at $T=3 \times 10^{5} \mathrm{~K}$ and in collisional ionization equilibrium, the OVII/O VI ratio is 1.7 , which leads to an expected contribution of $N_{\text {OVII }}=2-7 \times 10^{14} \mathrm{~cm}^{-2}$. In contrast, the typical O VII column (assuming an optically thin plasma) is $N_{\text {OVII }} \sim 4-5 \times 10^{15} \mathrm{~cm}^{-2}$ (Miller \& Bregman 2013). Hence, the contribution directly from O VI absorbers in the Galaxy is at most $15 \%$. It is likely lower, since Gupta et al. (2012) and Miller \& Bregman (2015) argue that the O VII lines are not optically thin. The bias from the $\mathrm{O}$ VI absorbers also declines at higher latitude, since the O VII column declines more slowly than the O VI column with increasing $b$ (corroborated in emission by Henley \& Shelton 2013). For most of our sight lines we estimate that the local and disk contribution to the 
O VII column is less than $10 \%$.

Even a $10 \%$ contribution is important for measuring the true halo velocity, but compared to the uncertainty in our best-fit velocity parameters, it is small. More importantly, as with the hot disk considered above, it cannot by itself account for the measured velocities if we assume that the million-degree halo is stationary.

\subsection{Summary and Conclusions}

We have measured a signature of rotation in the O VII line around the Milky Way using 37 sight lines towards background AGNs and archival RGS data. The parametric fit strongly depends on the brightest AGNs, but nonparametric tests indicate that the O VII absorbers are not stationary, even when removing suspect lines. From larger samples of emission and absorption lines, we believe that an extended halo is more consistent with the data than an exponential (thick) disk, and in this case this halo rotates at some velocity smaller than $v_{\mathrm{LSR}}$. Taken at face value, this implies that the million-degree gas has a comparable angular momentum to the galaxy disk. It is possible that both components exist, in which case the RGS lines are blends of kinematically distinct components. We use a large sample of emission lines to constrain the contribution of each component to the column density, and find that an exponential disk accounts for no more than $10 \%$ of the O VII column density. Thus, the measured Doppler shifts are dominated by the motion of the gas in the extended halo, which is consistent with prograde rotation. Even if a disk were to dominate (which, for our data, produces an unacceptable $\chi^{2}$ value), the best-fit azimuthal velocities imply that it is rotating at nearly the same speed and with a comparable amount of angular momentum to the spherical model.

This conclusion depends on assumptions about the underlying model. Measuring the true velocity and separating the X-ray absorbers into their various components requires a high-resolution X-ray instrument with a large effective area (e.g. ARCUS, Smith et al. 2014), which would also give important information on their line shapes that could constrain the optical depth and Doppler $b$ parameters (Miller et al. 2016), as well as reveal the contribution to the total column from the disk and the coronal halo gas seen in X-ray emission.

The recent work on calibrating the conversion of dispersion angle to a wavelength grid for the RGS (de Vries et al. 2015) and stacking multiple observations of the same object enables a wavelength accuracy of tens of $\mathrm{km} \mathrm{s}^{-1}$ for the first time (Figure 1). After investigating a variety of systematic issues, we find that the statistical uncertainty (due to the low $S / N$ in many spectra and the relatively small sample) remains the major source of error. The inferred halo velocity and angular momentum are strongly model dependent (and the uncertainties that we report are large), but the basic conclusion that the hot gas distribution rotates is less so.

Several scenarios could produce a rotating halo that lags behind the disk, depending on the geometry. These include a galactic fountain of cool gas that spins up hot gas (Marinacci et al. 2011), a hot galactic fountain of superbubble ejecta that produces an exponential disk before cooling, or infall from the cosmic web with some preferential direction. Our measurements cannot, by themselves, distinguish between these models (which may not be mutually exclusive), but they are an important kinematic constraint for future halo and galaxy formation models.

We thank Eric Bell for analysis suggestions and constructive criticism, and Oleg Gnedin for information on the Galaxy's angular momentum. Frits Paerels provided technical advice regarding the RGS. We thank the referees for substantial comments that significantly improved the manuscript. We gratefully acknowledge financial support from the NASA ADAP program, through grant NNX11AG55G.

\section{REFERENCES}

Anderson, M. E., \& Bregman, J. N. 2011, ApJ, 737, 22

Anderson, M. E., Bregman, J. N., \& Dai, X. 2013, ApJ, 762, 106

Arnaud, K. A. 1996, in Astronomical Society of the Pacific Conference Series, Vol. 101, Astronomical Data Analysis Software and Systems V, ed. G. H. Jacoby \& J. Barnes, 17

Bhattacharjee, P., Chaudhury, S., \& Kundu, S. 2014, ApJ, 785, 63

Bovy, J., Hogg, D. W., \& Rix, H.-W. 2009, ApJ, 704, 1704

Bowen, D. V., Jenkins, E. B., Tripp, T. M., et al. 2008, ApJS, 176,59

Bregman, J. N., \& Lloyd-Davies, E. J. 2007, ApJ, 669, 990

Brunthaler, A., Reid, M. J., Menten, K. M., et al. 2011, Astronomische Nachrichten, 332, 461

Cen, R. 2012, ApJ, 753, 17

Cox, D. P., \& Reynolds, R. J. 1987, ARA\&A, 25, 303

Crain, R. A., McCarthy, I. G., Frenk, C. S., Theuns, T., \& Schaye, J. 2010, MNRAS, 407, 1403

de Vries, C. P., den Herder, J. W., Gabriel, C., et al. 2015, A\&A, 573, A128

Drake, G. W. 1988, Canadian J. Phys., 66, 586

Faerman, Y., Sternberg, A., \& McKee, C. F. 2016, ArXiv e-prints, arXiv:1602.00689

Fang, T., Bullock, J., \& Boylan-Kolchin, M. 2013, ApJ, 762, 20

Fang, T., Buote, D. A., Bullock, J. S., \& Ma, R. 2015, ArXiv e-prints

Fang, T., Marshall, H. L., Lee, J. C., Davis, D. S., \& Canizares, C. R. 2002, ApJ, 572, L127

Fang, T., Mckee, C. F., Canizares, C. R., \& Wolfire, M. 2006, ApJ, 644, 174

Foster, A. R., Ji, L., Smith, R. K., \& Brickhouse, N. S. 2012, ApJ, 756, 128

Galeazzi, M., Chiao, M., Collier, M. R., et al. 2014, Nature, 512 171

Gupta, A., Mathur, S., Krongold, Y., Nicastro, F., \& Galeazzi, M. 2012, ApJ, 756, L8

Hagihara, T., Yamasaki, N. Y., Mitsuda, K., et al. 2011, PASJ, 63, S889

Hagihara, T., Yao, Y., Yamasaki, N. Y., et al. 2010, PASJ, 62, 723

Henley, D. B., \& Shelton, R. L. 2012, ApJS, 202, 14

-. 2013, ApJ, 773, 92

-. 2015, ApJ, 808, 22

Henley, D. B., Shelton, R. L., Kwak, K., Joung, M. R., \& Mac Low, M.-M. 2010, ApJ, 723, 935

Hill, A. S., Joung, M. R., Mac Low, M.-M., et al. 2012, ApJ, 750, 104

Huenemoerder, D. P., Mitschang, A., Dewey, D., et al. 2011, AJ 141,129

Joung, M. R., Putman, M. E., Bryan, G. L., Fernández, X., \& Peek, J. E. G. 2012, ApJ, 759, 137

Kalberla, P. M. W., Dedes, L., Kerp, J., \& Haud, U. 2007, A\&A, 469,511

Karataş, Y., Bilir, S., Eker, Z., \& Demircan, O. 2004, MNRAS, 349, 1069

Kuntz, K. D. 2009, in American Institute of Physics Conference Series, Vol. 1156, American Institute of Physics Conference Series, ed. R. K. Smith, S. L. Snowden, \& K. D. Kuntz, 3-15 Li, J.-T., \& Wang, Q. D. 2013, MNRAS, 435, 3071

Marinacci, F., Fraternali, F., Nipoti, C., et al. 2011, MNRAS, 415,1534 
McKernan, B., Yaqoob, T., \& Reynolds, C. S. 2004, ApJ, 617, 232

Miller, M. J., \& Bregman, J. N. 2013, ApJ, 770, 118

-. 2015, ApJ, 800, 14

Miller, M. J., Hodges-Kluck, E. J., \& Bregman, J. N. 2016, ArXiv e-prints, arXiv:1601.06797

Mo, H., van den Bosch, F. C., \& White, S. 2010, Galaxy Formation and Evolution

Nicastro, F., Senatore, F., Gupta, A., et al. 2016, MNRAS, 457, 676

Nicastro, F., Zezas, A., Drake, J., et al. 2002, ApJ, 573, 157

Nicastro, F., Zezas, A., Elvis, M., et al. 2003, Nature, 421, 719

Paerels, F. B. S., \& Kahn, S. M. 2003, ARA\&A, 41, 291

Rasmussen, A., Kahn, S. M., \& Paerels, F. 2003, in Astrophysics and Space Science Library, Vol. 281, The IGM/Galaxy

Connection. The Distribution of Baryons at $\mathrm{z}=0$, ed. J. L.

Rosenberg \& M. E. Putman, 109

Rasmussen, A. P., Kahn, S. M., Paerels, F., et al. 2007, ApJ, 656, 129

Reid, M. J., Menten, K. M., Brunthaler, A., et al. 2014, ApJ, 783, 130

Salem, M., Besla, G., Bryan, G., et al. 2015, ArXiv e-prints, arXiv:1507.07935

Savage, B. D., Sembach, K. R., Wakker, B. P., et al. 2003, ApJS, 146,125

Sembach, K. R., Wakker, B. P., Savage, B. D., et al. 2003, ApJS, 146,165

Shapiro, P. R., \& Field, G. B. 1976, ApJ, 205, 762
Shelton, R. L., Sallmen, S. M., \& Jenkins, E. B. 2007, ApJ, 659, 365

Smith, R. K., Ackermann, M., Allured, R., et al. 2014, in Society of Photo-Optical Instrumentation Engineers (SPIE) Conference Series, Vol. 9144, Society of Photo-Optical Instrumentation Engineers (SPIE) Conference Series, 4

Snowden, S. L., Heiles, C., Koutroumpa, D., et al. 2015, ApJ, 806,119

Snowden, S. L., Egger, R., Freyberg, M. J., et al. 1997, ApJ, 485, 125

Wakker, B. P., Savage, B. D., Sembach, K. R., et al. 2003, ApJS, 146,1

Wang, Q. D., Yao, Y., Tripp, T. M., et al. 2005, ApJ, 635, 386

Welsh, B. Y., \& Shelton, R. L. 2009, Ap\&SS, 323, 1

White, S. D. M., \& Frenk, C. S. 1991, ApJ, 379, 52

Williams, R. J., Mathur, S., \& Nicastro, F. 2006, ApJ, 645, 179

Williams, R. J., Mathur, S., Nicastro, F., \& Elvis, M. 2007, ApJ, 665,247

Williams, R. J., Mathur, S., Nicastro, F., et al. 2005, ApJ, 631, 856

Xue, X. X., Rix, H. W., Zhao, G., et al. 2008, ApJ, 684, 1143

Yao, Y., Tripp, T. M., Wang, Q. D., et al. 2009a, ApJ, 697, 1784

Yao, Y., \& Wang, Q. D. 2005, ApJ, 624, 751

—. 2007, ApJ, 658, 1088

Yao, Y., Wang, Q. D., Hagihara, T., et al. 2009b, ApJ, 690, 143 